\title{
Unifying Recursive and Co-recursive Definitions in Sheaf Categories ${ }^{\star}$
}

\author{
Pietro Di Gianantonio and Marino Miculan \\ Dipartimento di Matematica e Informatica, Università di Udine, \\ Via delle Scienze 206, 33100 Udine, Italy. \\ \{digianantonio,miculan\}@dimi.uniud.it
}

\begin{abstract}
In this paper we present a theorem for defining fixed-points in categories of sheaves. This result gives a unifying and general account of most techniques used in computer science in order to ensure convergency of circular definitions, such as (but not limited to) well-founded recursion and contractivity in complete ultra metric spaces. This general fixed-point theorem encompasses also a similar set theoretic result presented in previous work, based on the notion of ordered family of equivalences, and implemented in the Coq proof assistant.
\end{abstract}

\section{Introduction}

Circular definitions are pervasive and fundamental in many fields of Mathematics and Computer Science. However, it is well known that not all circular definitions are meaningful, i.e. converging. Many different criteria and techniques have been introduced for establishing when a circular definition is well given, and in this case to calculate what is its meaning - the fixed point.

One approach is to look for syntactically decidable criteria for recognizing well-given circular definition. For defining objects in inductive datatypes we have thus, beside the traditional iteration and recursion schemata, various criteria such as the guarded by destructors condition (adopted in Coq [138]). For defining objects in coinductive datatypes we have several coiteration and corecursion schemata, and the guarded by constructors condition of Coq [6]. These syntactic, intensional criteria can be completely automatized in order to mechanically check that a given definition is correct. However, syntactic criteria have always a limited expressive power, and many sound definitions are rejected.

In order to overcome this expressivity limitation, another approach is to establish general results about the existence of fixed points in suitable semantic domains. In this case, a well-formed circular definition comes equipped with a formal assessment of its convergency, according to the theoretical properties of the intended model. A well-known and very general method for building models supporting recursive definitions is well-founded recursion (and variations thereof) 12,1,2, . A formal definition of a function by well-founded recursion must contain also the definition of the order over which the recursion goes, and the proof

\footnotetext{
* Work supported by italian MIUR project COFIN 2001013518 CoMETA.
} 
that the order is well-founded. In this case, the model is specified by the wellfounded order, and a general result ensures the existence of the unique function recursively defined.

Another important method for constructing models supporting circular definitions is based on the Banach fixed point theorem for complete (ultra)metric spaces. In this case, the fixed point of a (contractive) function $f: D \rightarrow D$ is obtained by starting from an arbitrary point $x \in D$, and iterating the $f$ function $\omega$ times; this leads to a sequence

$$
x, f(x), f^{2}(x), f^{3}(x), \ldots
$$

whose limit $x_{\omega}$, which exists by completeness, is the desired fixed point. Despite its proved usefulness in many fields (e.g., in concurrency theory [3]), Banach fixed point theorem has some limitations. First of all, one can find several examples in Computer Science and Mathematics where the fixed point of a function cannot be reached simply by this construction, but in which it is necessary to go beyond $\omega$ iterations, e.g. to construct a chain

$$
\ldots, x_{\omega}, f\left(x_{\omega}\right), f^{2}\left(x_{\omega}\right), f^{3}\left(x_{\omega}\right), \ldots x_{2 \omega}, f\left(x_{2 \omega}\right), f^{2}\left(x_{\omega}\right), \ldots, x_{\omega^{2}}, \ldots, x_{\omega^{\omega}} \ldots
$$

by repeatedly applying the function $f$ and limit construction, until a fixed point is eventually reached. This sort of transfinite constructions cannot be accommodated inside a complete ultra metric space structure.

Another striking limitation is that in these approaches, the realm of "recursive" definitions in inductive spaces, and the realm of "co-recursive" definitions in co-inductive spaces are kept well apart: well-founded induction applies only to recursive definition and Banach fixed point theorem applies only to co-recursive definition. However, it is common in Computer Science to face mixed recursivecorecursive definitions, i.e., definitions whose soundness relies on both recursive and co-recursive arguments at once (see [4] for examples). Neither the sole Banach fixed point theorem, nor the mere well-founded induction principles are enough for dealing with these mixed definitions.

Therefore, what we need is a more general class of models, unifying the approach based on Banach fixed point theorem in (ultra)metric spaces and that based on the well founded induction principle, with a general result for establishing fixed points of circular definitions. This result should support "mixed" definitions, with both recursive and co-recursive aspects at once, and possibly with transfinite fixpoint constructions. This is the subject of this paper.

A first step in this direction has been made in [4, where we introduced a fixed point theorem which can be used to prove the convergency of mixed recursive/corecursive definition. This theorem is a generalization of the Banach fixed point theorem on ultra-metric space, with the idea of allowing the construction of a fixed point by iteration beyond the cardinal $\omega$. An aim of [4] was to give a result which can be formalized and used easily inside a logical framework (e.g., Coq, or Isabelle as in [11]), in order to prove the existence and to effectively construct fixed points of functions. For this purpose, our theorem had to be constructive, and to deal with very simple structures. This simplicity is useful because in order 
to apply the theorem in the proof editor (Coq, in this case) on a function $f$ defined on a structure $X$, we need to prove explicitly that $X$ is a instance of our generalized complete ultrametric space and that the function $f$ is indeed contractive. In order to keep this overhead as low as possible, we looked for a minimal set of relevant and simple features of generalized complete ultrametric spaces and contractive functions. Thus, in 4] we come to the set theoretic notion of (complete) ordered family of equivalences, which can be seen as a generalization and a simplification at once of the notion of complete ultra metric space. Over c.o.f.e.'s we defined a natural and general notion of contractivity, encompassing most schemata usually adopted for ensuring soundness of circular definitions. The conditions that a space has to satisfy in order to be a c.o.f.e. are simple and direct, hence the burden on the user for applying the fixed point theorem is limited.

One natural question arising from this previous work is whether the conditions presented in [4] (and the similar ones in 11]) were somehow arbitrary, or they can be explained within a more general setting - and possibly generalized further. In this work, we answer positively to this question. We analyze these constructions using categorical-theoretic tools, namely sheaf categories. Quite surprisingly, it comes out that the completeness conditions that we defined in [4] are almost equivalent to the amalgamation condition on sheaves, and therefore that our generalized ultra metric spaces can be seen as a particular kind of sheaves. Moreover, the set-theoretic fixed point construction by well-founded induction of [4] can be extended to more general sheaves over a topology satisfying a sort of well-founded condition.

In summary, the results of this work are twofold. First, we give a sheaftheoretic explanation of the theory developed in [4], given by a correspondence between the completeness of o.f.e.'s and the amalgamation condition of sheaves. Moreover, in this categorical setting we can generalize further our previous fixed point theorem. This leads for instance to a more elegant treatment of one of the leading applications, i.e. the definition of functions by well-founded recursion.

Synopsis. In Section [2 we recall the basic definitions about topological spaces, and presheaves and sheaves over topological spaces. In Section 3 we develop a general theory of fixed points in sheaf categories, unifying inductive and coinductive aspects of circular definitions. The connections between the categoricaltheoretic results of this work, and the set-theoretic ones presented in previous work (and implemented in Coq) is described in Section 4 . Final conclusions and future work are discussed in Section 5 .

\section{Basic Definitions}

\subsection{Topological Spaces}

Recall that a topological space is a pair $(X, \mathcal{O}(X))$ where $X$ is a set and $\mathcal{O}(X)$, the topology over $X$, is a subset of $\wp(X)$ closed by arbitrary union and finite intersection, and $\emptyset, X \in \mathcal{O}(X)$. We often denote a topological space by its topology. We assume the reader familiar with the basic notions and properties from topology theory; see e.g. [9]. 
Definition 1. A base for a topological space $(X, \mathcal{O}(X))$ is a family $\mathcal{K} \subseteq \mathcal{O}(X)$ of open sets, ranged over by $K$, such that for every $V \in \mathcal{O}(A)$ there exists a family $\left(K_{i}\right)_{i \in I}$ of elements of the base, called a covering of $V$, such that $V=\cup_{i \in I} K_{i}$.

A particular example of topological space that we will use quite often is the following:

Definition 2. Let $(A,<)$ be an order. We defined the downward closed topology over $A$, denoted by $\mathcal{O}(A)$, as the one whose open sets are all downward closed sets: for $U \subseteq A, U \in \mathcal{O}(A)$ if and only if for all $a \in U$, if $b<a$ then $b \in U$.

It is immediate to see that $\mathcal{O}(A)$ is closed under arbitrary intersection and union, thus $\mathcal{O}(A)$ is indeed a topology. The smallest base for the downward closed topology is the set of cones on $(A,<)$, that is the sets of the form $\downarrow a \triangleq\left\{a^{\prime} \mid\right.$ $\left.a^{\prime}<a\right\} \cup\{a\}$. In the following the set of cones of $A$ will be denoted by $\mathcal{K}_{A}$.

In several example we will consider the set $\omega$ of the natural numbers together with the standard order. It is immediate to see that the open sets of the downward closed topology over $\omega$ are cones. In particular, by the standard construction of defining an ordinal number as the set of its predecessor, we have that $\mathcal{O}(\omega)=\omega+1$. In other words, the elements of $\mathcal{O}(\omega)$ are the natural numbers and the set $\omega$ itself.

\section{$2.2 \quad$ Presheaves}

Any topology $\mathcal{O}(X)$ with the subset relation forms an order (a complete Heyting algebra, actually). Therefore, as usual in category theory, we can see $\mathcal{O}(X)$ as a category with exactly one morphism $i n_{U, V}: U \rightarrow V$ whenever $U \subseteq V$.

Definition 3. A presheaf over (the topology) $\mathcal{O}(X)$ is a functor $P: \mathcal{O}(X)^{o p} \rightarrow$ $\mathcal{S}$ et. Presheaves and natural transformations among them are objects and morphisms of the functor category $\widehat{\mathcal{O}(X)} \triangleq \mathcal{S} e t^{\mathcal{O}(X)^{o p}}$.

In particular we consider the following running examples:

Example 1. (a) (Partial functions) A classical example of presheaf is the one formed by sets of partially defined continuous functions on a topological set. Let $\mathcal{O}(X), \mathcal{O}(Y)$ be two topological spaces. The presheaf $F_{X, Y}: \mathcal{O}(X)^{o p} \rightarrow$ $\mathcal{S}$ et of partial continuous functions to $Y$ is defined on objects as follows:

$$
F_{X, Y}(U) \triangleq U \rightarrow Y
$$

On morphisms, $F_{X, Y}$ is defined by function restriction: given a morphism $i n_{U, V}: U \rightarrow V$ in $\mathcal{O}(X)$ (i.e. $U \subseteq V$ ), and a continuous function $f: V \rightarrow Y$, then

$$
\left.F_{X, Y}\left(i n_{U, V}\right)(f) \triangleq f\right|_{U}
$$

i.e. the restriction of $f$ to the elements of $U$. 
(b) (Ultra-metrics) Recall that an ultra-metric space $S$ is a metric space whose distance $d:(S \times S) \rightarrow \mathbb{R}$ satisfies a stronger version of the triangular inequality, namely for all $r, s, t \in S: d(r, s) \leq \max \{d(r, t), d(t, s)\}$. As a consequence, balls in an ultra-metric space are equivalence classes. An ultrametric space $S$ together with a real number $c \in(0,1)$ induce a presheaf $M_{S, c}: \mathcal{O}(\omega)^{o p} \rightarrow \mathcal{S}$ et, defined on objects by $M_{S, c}(\alpha) \triangleq S / \equiv_{\alpha}$, where $\equiv_{\alpha}$ is the equivalence on $S$ defined by

$$
s \equiv_{\alpha} t \stackrel{\triangle}{\Longleftrightarrow} \forall n \in \alpha \cdot d(s, t) \leq c^{n}
$$

or, more concretely,

$$
s \equiv_{n+1} t \Longleftrightarrow d(s, t) \leq c^{n} \quad s \equiv_{\omega} t \Longleftrightarrow s=t .
$$

On morphisms $M_{S, c}$ is defined by class immersion: given a morphism $i n_{\alpha, \alpha^{\prime}}$ in $\mathcal{O}(\omega)$ we define

$$
M_{S, c}\left(i n_{\alpha, \alpha^{\prime}}\right)\left([s]_{\alpha^{\prime}}\right)=[s]_{\alpha}
$$

(c) (Ordered families of equivalence) Recall from 4 that an ordered family of equivalences (o.f.e.) is a tuple $\mathcal{O}=\langle A,<, X, \equiv\rangle$ where $A$ (the carrier) and $X$ (the domain) are sets, $<$ is a well-founded order on $A$ and $\equiv$ is an $A$ indexed family of equivalence relations $\left\{\equiv_{a}\right\}_{a \in A}$ on $X$. If $U \subseteq A$, we define the equivalence relation $\equiv_{U}$ on $X$ as

$$
x \equiv_{U} y \stackrel{\triangle}{\Longleftrightarrow} \forall a \in U . x \equiv_{a} y
$$

Along the same lines of the previous example, given an o.f.e. $(A,<, X, \equiv)$ one can define a presheaf $E_{A, X}: \mathcal{O}(A)^{o p} \rightarrow \mathcal{S}$ et, formed by equivalence classes, as $E_{S, c}(U) \triangleq X / \equiv_{U}$. On morphisms $E_{A, X}$ is defined by class immersion in the same way of $M_{S, c}$.

Notation. Let $P: \mathcal{O}(X)^{o p} \rightarrow \mathcal{S}$ et be a presheaf, and $i n_{U, V}: U \rightarrow V$ a morphism between two open sets of the topology (i.e. an inclusion). For $a \in P_{V}$, the element $P_{i n_{U, V}}(a) \in P_{U}$ will be denoted by $\left.a\right|_{U}$ as a syntactic shorthand.

\subsection{Sheaves}

We consider now the category of sheaves $\mathrm{Sh}(A)$ over a topological space $(A, \mathcal{O}(A))$, as defined in [10, II.10].

Definition 4. A presheaf $P: \mathcal{O}(X)^{o p} \rightarrow \mathcal{S}$ et is called a sheaf if it satisfies the following property: for all $U \in \mathcal{O}(X)$, for all open covering $\left\{U_{i}\right\}_{i \in I}$ of $U$ (that is $\left.U=\bigcup_{i \in I} U_{i}\right)$, and for all sections $\left\{s_{i} \in P_{U_{i}}\right\}_{i \in I}$ pairwise compatible (that $i s$, for all $i, j \in I:\left.s_{i}\right|_{U_{i} \cap U_{j}}=\left.s_{j}\right|_{U_{i} \cap U_{j}}$ ), there exists a unique $s \in P_{U}$ (called the amalgamation) such that for all $i \in I:\left.s\right|_{U_{i}}=s_{i}$.

The full subcategory of $\widehat{\mathcal{O}(A)}$ whose objects are sheaves is denoted by $\operatorname{Sh}(A)$. 
By applying this definition to the three running examples we have:

Example 2. (a) (Partial functions) For any two topological spaces $\mathcal{O}(X), \mathcal{O}(Y)$, the presheaf $F_{X, Y}$ is always a sheaf. In fact given an open set $U \in \mathcal{O}(X)$, and an open covering $\left\{U_{i}\right\}_{i \in I}$ of $U$, a family of sections $\left\{\left[f_{i}\right] \in F_{X, Y}\left(U_{i}\right)\right\}_{i \in I}$ is pairwise compatible if for every pair $i, j \in I$ we have $\forall x \in U_{i} \cap U_{j} \cdot f_{i}(x)=$ $f_{j}(x)$. The amalgamation $[f]$ of this family can be defined as

$$
f(x) \triangleq f_{i}(x) \text { with } x \in U_{i}
$$

It is immediate to check that the definition is independent from the choice of $i$ and therefore correct.

(b) (Ultra-metrics) The presheaf $M_{S, c}$ is a sheaf if and only if the space $S$ is complete as a metric space. In fact, given a complete metric space $S$, a cardinal $\alpha \in \mathcal{O}(\omega)$, and a set $\left\{n_{i}\right\}_{i \in I}$ of cardinals having $\alpha$ as lub (i.e., a covering of $\alpha$ ), the sections $\left\{\left[s_{i}\right]\right\}_{i \in I}$ in $M_{S, c}$ are pairwise compatible if for every pair $i, j \in I$, whenever $n_{i} \leq n_{j}$ we have that $d\left(s_{i}, s_{j}\right) \leq c^{n_{i}-1}$. If $\alpha$ is a natural number then there exists $n_{i}=\alpha$ and the amalgamation point can be readily defined as $s=s_{i}$. If $\alpha=\omega$ then one can choose an increasing sequence of naturals $n_{k_{0}}<n_{k_{1}}<n_{k_{2}}<\ldots$; it is then immediate to check that $s_{n_{k_{0}}}, s_{n_{k_{1}}}, s_{n_{k_{2}}}, \ldots$ is a Cauchy sequence whose limit (which exists by completeness) is the amalgamation point.

The proof of the other implication, namely that if $M_{S, c}$ is a sheaf then $S$ is a complete metric space, is almost immediate.

(c) (Complete ordered families of equivalence) Given an ordered family of equivalences $(A,<, X, \equiv)$, the presheaf $E_{A, X}$ is a sheaf if and only if the order family of equivalence $(A,<, X, \equiv)$ is complete, in the sense of [4]. We will examine the connection between complete o.f.e.'s and sheaves in more detail in Section 4.2 below.

\section{Fixed Points in Sheaf Categories}

In this section, we present a general result for defining fixed points in sheaves. Our aim is to unify the coinductive approach, typical of complete metric spaces, and the inductive approach of definitions over well-founded orders. The amalgamation condition of sheaves provides what is needed for dealing with coinductive parts; on the other hand, the inductive counterparts corresponds to requiring a well-founded condition on the topology:

Definition 5. A base $\mathcal{K}$ for a topological space $(X, \mathcal{O}(X))$ is said well-founded if $(\mathcal{K}, \subseteq)$ is a well-founded order, i.e., there exists no succession of base elements $K_{1}, K_{2}, K_{3}, \ldots \in \mathcal{K}$ such that $K_{1} \supset K_{2} \supset K_{3} \ldots$

A topology is well-founded if it has a well-founded base.

In particular, an order $(A,<)$ is well-founded if and only if the downward closed topology on $A$ is well-founded. Indeed, the set of cones $\mathcal{K}_{A}$ on $A$ form a minimal base for $\mathcal{O}(A)$; therefore, $\mathcal{O}(A)$ is well founded if and only if $\mathcal{K}_{A}$ is, and moreover 
the well-founded condition on $\mathcal{K}_{A}$ is immediately equivalent to the well-founded condition for $(A,<)$.

An example of not well-founded topology is the real line $\mathbb{R}$ with the Euclidean topology. Let $\mathcal{K}$ be any basis for $\mathbb{R}$, and $K \in \mathcal{K}$. Let us consider any open interval $U \subseteq K$, and let $V$ be the open interval formed by the first half of $U$; clearly $V$ is open, and therefore there exists $K^{\prime} \in \mathcal{K}$ such that $K^{\prime} \subseteq V \subset U \subseteq K$. Repeating this procedure, we can define a non well-founded chain $K \subset K^{\prime} \subset K^{\prime \prime} \ldots$

In order to define a notion of contractivity for morphisms on (pre)sheaves we need to introduce the following definition:

Definition 6. Let $\mathcal{K}$ be a base for a topological space $(X, \mathcal{O}(X))$. We define the predecessor operator $\mathrm{p}: \mathcal{O}(X) \rightarrow \mathcal{O}(X)$ as follows

$$
\mathrm{p} U \triangleq \bigcup\{K \in \mathcal{K} \mid K \subsetneq U\}
$$

The operator $\mathrm{p}$ is clearly monotone so it can be seen as a functor on $\mathcal{O}(X)$. Moreover, $\mathrm{p}$ is decreasing (for all $U \in \mathcal{O}(X): \mathrm{p} U \subseteq U$ ), so there exists a (unique) inclusion natural transformation $\mathrm{i}: \mathrm{p} \longrightarrow I d$.

Note that $\mathrm{p}$ differs from the identity only on the elements of the basis and that in the topology $\mathcal{O}(\omega)$ we have that $\mathrm{p} n=n-1$ and $\mathrm{p} \omega=\omega$.

In the following, for $P: \mathcal{O}(X)^{o p} \rightarrow \mathcal{S}$ et, we will denote by $P$ p : $\mathcal{O}(X)^{o p} \rightarrow \mathcal{S}$ et the presheaf obtained by composition, i.e. $(P \mathrm{p})_{U}=P_{\mathrm{p} U}$ and for $i n_{U, V}: U \rightarrow V$, $(P \mathrm{p})_{i n_{U, V}}=P_{\mathrm{p}_{i n}, V}: P_{\mathrm{p} V} \rightarrow P_{\mathrm{p} U}$. Similarly, $P \mathrm{i}$ is the natural transformation $P \mathrm{i}: P \longrightarrow P \mathrm{p}$ defined by componentwise application: for $U \in \mathcal{O}(X),(P \mathrm{i})_{U}=$ $P_{\mathrm{i}_{U}}=P_{i n_{\mathrm{p} U, U}}: P_{U} \rightarrow P_{\mathrm{p} U}\left(\right.$ since $\left.\mathrm{i}_{U}=i n_{\mathrm{p} U, U}: \mathrm{p} U \rightarrow U\right)$.

Definition 7. Given a well-founded base $\mathcal{K}$ for the topology $\mathcal{O}(X)$ and a presheaf $P: \mathcal{O}(X)^{o p} \rightarrow \mathcal{S}$ et, a natural transformation $f: P \longrightarrow P$ is contractive (w.r.t. $\mathcal{K}$ ) if it factorizes along the natural transformation $P \mathrm{i}: P \longrightarrow P \mathrm{p}$, i.e., there exists a natural transformation $\bar{f}: P \mathrm{p} \longrightarrow P$ such that $f=\bar{f} \circ P \mathrm{i}$ :
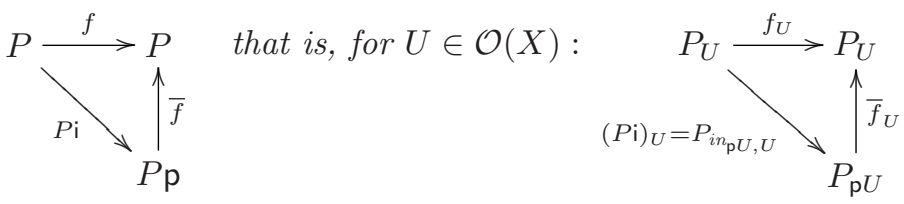

It is interesting to observe that the above definition of contractivity encompasses most standard and well-known criteria used for ensuring existence of a fixed point, such as the "guarded by constructors/destructors" conditions. More generally, these criteria are subsumed by the following examples:

Example 3. (a) (Recursive definitions of functions) Given a well-founded relation $<$ on a set $A$ and $a \in A$, let us denote with $\downarrow a$ the set $\rfloor a \triangleq\{b \mid b<a\}$. Then a recursive definition of a function $A \rightarrow B$ is given by a function $G$ which maps an element $a \in A$ and a function $f: \downarrow a \rightarrow B$ to a value in $B \square$

${ }^{1}$ Using dependent types, the arity of $G$ is $G: \prod_{a \in A}(\downarrow a \rightarrow B) \rightarrow B$. 
In this setting the function $h: A \rightarrow B$ recursively defined by $G$ is the unique function satisfying the equation:

$$
h(a)=G\left(a,\left.h\right|_{\downarrow a}\right)
$$

Let consider the downward closed topology, $\mathcal{O}(A)$, on the partial order $A$ and the coarsest topology on $B$, i.e. the topology having as open sets just the empty set and total space $B$. Given these two topological spaces, we can consider the sheaves of partial continuous $F_{A, B}$ and we have that the function $G$ induces a natural transformation $G^{*}: F_{A, B} \longrightarrow F_{A, B}$ in $\operatorname{Sh}(A)$ defined as follows:

$$
G_{U}^{*}(f) \triangleq \lambda a \cdot G\left(a,\left.f\right|_{\downarrow a}\right)
$$

It is straightforward to prove that $G^{*}$ is indeed a natural transformation. Moreover, $G^{*}$ is contractive w.r.t. the base $\mathcal{K}_{A}$, formed by the cones of $A$ : indeed, we can define $\overline{G^{*}}$ by

$$
{\overline{G^{*}}}_{U}\left(\left.f\right|_{\mathrm{p} U}\right) \triangleq \lambda a \cdot G\left(a,\left.\left(\left.f\right|_{\mathrm{p} U}\right)\right|_{\downarrow a}\right)
$$

The above definition is correct since for each $U \in \mathcal{O}(A)$ and $a \in U$ we have that $\rfloor a \subseteq \mathrm{p} U$ and therefore $\left.\left.f\right|_{\mathrm{p} U}\right)\left.\right|_{\downarrow a}$ is well defined.

(b) (Ultrametrics) Let $(S, d:(S \times S) \rightarrow[0,1])$ be an ultrametric space, and $f: S \rightarrow S$ a function contractive with constant $c \in[0,1)$ (in the metric $d$ ). Then, $f$ induces a natural transformation $f^{*}: M_{S, c} \longrightarrow M_{S, c}$ given by

$$
f_{U}^{*}\left([s]_{U}\right) \triangleq[f(s)]_{U}
$$

Clearly $f^{*}$ is well-defined, and moreover contractive in the sense of Definition 7): the factorizing natural transformation $\overline{f^{*}}$ is given by

$$
\overline{f *}_{U}\left([s]_{\mathrm{p} U}\right)=[f(s)]_{U}
$$

The contractivity condition on the function $f$ ensures that this definition is correct, i.e. it preserves equivalences. Quite obviously $\overline{f^{*}}$ satisfies the required factorization.

(c) (Ordered families of equivalence) Using the same pattern of the two previous example we can reduce the notion of contractivity on o.f.e.'s [4, Definition 5], to the contractivity on presheaves of Definition [7. We will examine in detail this case in Section 4.1 below.

(Example 3)

Theorem 1 (General Fixed Point Theorem). Let $A$ be a topology with a well-founded base $\mathcal{K}$, and $P: \operatorname{Sh}(A)$ a sheaf on $A$. Then, every natural transformation $f: P \longrightarrow P$ contractive w.r.t. $\mathcal{K}$ has a unique fixed point, i.e. there exists a unique natural transformation $\mu: 1 \longrightarrow P$ in $\operatorname{Sh}(A)$ such that $f \circ \mu=\mu$.

Proof. In order to define $\mu=\left\{\mu_{U}: 1 \rightarrow P_{U} \mid U \in \mathcal{O}(A)^{o p}\right\}$, it suffices to define only the components on base elements, $\left\{\mu_{K} \in P_{K} \mid K \in \mathcal{K}\right\}$, and prove that they satisfy the naturality condition, i.e., for all $K, K^{\prime} \in \mathcal{K}$,

$$
\text { if } K^{\prime} \subseteq K \text { then }\left.\mu_{K}\right|_{K^{\prime}}=\mu_{K^{\prime}}
$$


In virtue of this fact we can then extend by amalgamation the definition of $\mu$ to any open set $U$. If $U=\cup_{i \in I} K_{i}$, then $\mu_{U} \in P_{U}$ is defined as the amalgamation of the sections $\left\{\mu_{K_{i}} \in P_{K_{i}} \mid i \in I\right\}$, which are pairwise compatible by (1).

We will define the components $\mu_{K}$ and prove the equality (1) at the same time, by well founded induction on the subset relation on the elements of the base $\mathcal{K}$. The definition of $\mu$ on elements of the base is the following:

$$
\mu_{K} \triangleq \bar{f}_{K} \circ \mu_{\mathrm{p} K}
$$

where $\mu_{\mathrm{p} K}: 1 \rightarrow P_{\mathrm{p} K}$ is defined as the amalgamation of the morphisms $\left\{\mu_{K^{\prime}}\right.$ : $\left.1 \rightarrow P_{K}^{\prime} \mid K^{\prime} \subsetneq K\right\}$. The equality (1) is proved by observing that the following diagram commutes:

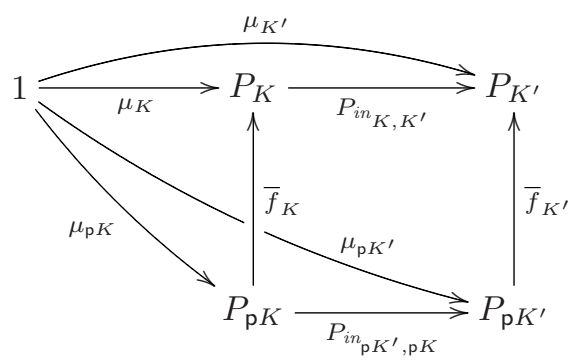

In fact, $\mu_{K}=\bar{f}_{K} \circ \mu_{\mathrm{p} K}$ and $\mu_{K^{\prime}}=\bar{f}_{K^{\prime}} \circ \mu_{\mathrm{p} K^{\prime}}$ by definition, while the square commutes by naturality of $\bar{f}$. It remains to prove that $P_{i n_{\mathrm{p} K^{\prime}, \mathrm{p} K}} \circ \mu_{\mathrm{p} K}=\mu_{\mathrm{p} K^{\prime}}$, i.e. that $\left.\mu_{\mathrm{p} K}\right|_{\mathrm{p} K^{\prime}}=\mu_{\mathrm{p} K^{\prime}}$. Since for all $K^{\prime \prime} \subsetneq K^{\prime}$ we have that $\left.\left(\left.\mu_{\mathrm{p} K}\right|_{\mathrm{p} K^{\prime}}\right)\right|_{K^{\prime \prime}}=$ $\left.\mu_{\mathrm{p} K}\right|_{K^{\prime \prime}}=\mu_{\mathrm{p} K^{\prime}}$ it follows that $\left.\mu_{\mathrm{p} K}\right|_{\mathrm{p} K^{\prime}}$ is the amalgamation point of the elements $\left\{\mu_{K^{\prime \prime}} \mid K^{\prime \prime} \subsetneq K^{\prime}\right\}$ and therefore equal to $\mu_{\mathrm{p} K^{\prime}}$.

In order to prove the fixed-point equality $f \circ \mu=\mu$ it is sufficient to prove that it holds on the element of the base. Indeed, for each $K \in \mathcal{K}$ the following diagram commutes:

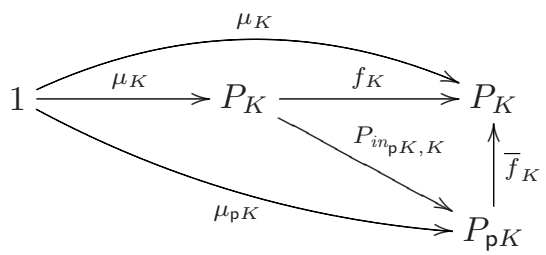

in fact by equality (11) $P i n_{\mathrm{p} K, K} \circ \mu_{K}$ is the amalgamation point of the morphisms $\left\{\mu_{K^{\prime}} \mid K^{\prime} \subsetneq K\right\}$ and therefore $\operatorname{Pin}_{\mathrm{p} K, K} \circ \mu_{K}=\mu_{\mathrm{p} K}$, while $f_{K}=\bar{f}_{K} \circ P i n_{\mathrm{p} K, K}$, by contractivity of $f$.

Given any other fixed-point $\rho$, the following diagram commute

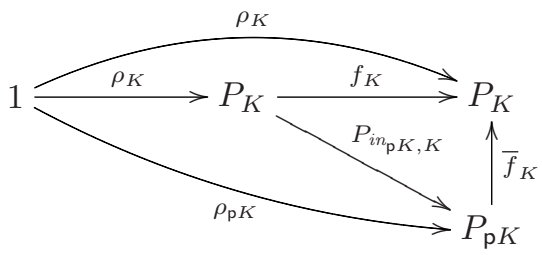


because $\rho_{K}=f_{K} \circ \rho_{K}$ by hypothesis, while $f_{K}=\bar{f}_{K} \circ P_{i n_{\mathrm{p} K, K}}$, by contractivity of $f$ and $P_{i n_{\mathrm{p} K, K}} \circ \rho_{K}=\rho_{\mathrm{p} K}$ by naturality of $\rho$.

Now we can finally prove, by inductions on the subset relation on the elements of the base, that for all $K \in \mathcal{K} \mu_{K}=\rho_{K}$. In fact by inductive hypothesis for all $K^{\prime} \subset K \mu_{K^{\prime}}=\rho_{K^{\prime}}$, so $\mu_{\mathrm{p} K}=\rho_{\mathrm{p} K}$, since both arrows are amalgamation points of equal sections. From this, by the last two diagrams, we have the thesis.

\section{Ordered Families of Equivalences and Presheaves}

In previous work [4], we have introduced and studied the notion of (complete) ordered families of equivalences, with the aim of developing a constructive, settheoretic approach (implemented in Coq) allowing for defining general fixed point by mixed inductive/coinductive definitions. In this section, we develop a categorical account of (complete) o.f.e.'s, and relate them to the theory developed in (pre)sheaf categories in Section 3

\subsection{Relating o.f.e.'s to Presheaves}

In Example 1(c), we have recalled the definition of ordered family of equivalences, and shown that any o.f.e. can be seen as a presheaf. More precisely, we show now that the category of presheaves over $A$ is connected to the category of o.f.e.'s by an adjunction.

Definition 8. Let $(A,<, X, \equiv),\left(A,<, X^{\prime}, \equiv^{\prime}\right)$ be two o.f.e.'s on the same order $(A,<)$. A morphism $f:(A,<, X, \equiv) \rightarrow\left(A,<, X^{\prime}, \equiv^{\prime}\right)$ is a function $f: X \rightarrow X^{\prime}$ such that for all $x, y \in X$, for all $a \in A$, if $x \equiv_{a} y$ then $f(x) \equiv_{a}^{\prime} f(y)$. The o.f.e.'s over $(A,<)$ and their morphisms form a category denoted by $\mathcal{O} f e(A,<)$.

The definition can be generalized further. A morphism between two o.f.e.'s on possibly different orders, is given by a pair $(h, f):(A,<, X, \equiv) \rightarrow\left(A^{\prime},<^{\prime}, X^{\prime}, \equiv^{\prime}\right)$ such that $h:\left(A^{\prime},<^{\prime}\right) \rightarrow(A,<)$ is monotone and $f: X \rightarrow X^{\prime}$ is such that for all $x, y \in X$, for all $a \in A$, if $x \equiv_{h a^{\prime}} y$ then $f(x) \equiv_{a^{\prime}}^{\prime} f(y)$. This gives rise to the category $\mathcal{O} f e$ of all ofe's over any well-founded order, which is a fibred category over the category $\mathcal{W}$ fo of well-founded orders. However, in the following we will dwell only in a fiber of this category at once, i.e., we will consider only the more restricted definition of morphisms.

We can define a functor $F: \mathcal{O} f e(A,<) \longrightarrow \widehat{\mathcal{O}(A)}$ as follows:

- for $O=(A,<, X, \equiv)$ an o.f.e., and $U \in \mathcal{O}(A)$, let $\left(F_{O}\right)_{U} \triangleq X / \equiv_{U}$, where the equivalence relation $\equiv_{U}$ over $X$ is defined as

$$
x \equiv_{U} y \stackrel{\triangleq}{\Longleftrightarrow} \forall a \in U: x \equiv_{a} y .
$$

For $i n_{U, V}: U \rightarrow V$ a (unique) morphism in $\mathcal{O}(A)$, we define $F_{O}\left(i n_{U, V}\right)$ : $X / \equiv_{V} \rightarrow X / \equiv_{U}$ as $F_{O}\left(i n_{U, V}\right)\left([x]_{\equiv_{V}}\right) \triangleq[x]_{\equiv_{U}}$. (In the following, for sake of simplicity, we will denote $[x]_{\equiv_{U}}$ just by $[x]_{U}$ ). 
- for $O=(A,<, X, \equiv), O^{\prime}=\left(A,<, X^{\prime}, \equiv^{\prime}\right)$ and $f: O \rightarrow O^{\prime}$ in $\mathcal{O} f e(A,<)$, we define the natural transformation $F_{f}: F_{O} \longrightarrow F_{O}$ whose component $\left(F_{f}\right)_{U}: X / \equiv_{U} \rightarrow X^{\prime} / \equiv_{U}^{\prime}$ is defined by $\left(F_{f}\right)_{U}\left([x]_{\equiv_{U}}\right) \triangleq[f(x)]_{\equiv_{U}^{\prime}}$.

The definition of the functor $G: \widehat{\mathcal{O}(A)} \longrightarrow \mathcal{O} f e(A,<)$ is as follows:

- the action of $G$ on the object $P$ is defined as $G_{P} \triangleq\left(A,<, P_{A}\right.$, $\left.\equiv\right)$, where for all $a \in A: x \equiv_{a} y \stackrel{\triangle}{\Longleftrightarrow} P_{i n_{\downarrow a, A}}(x)=P_{i n_{\downarrow a, A}}(y)$, ie if $\left.x\right|_{\downarrow a}=\left.y\right|_{\downarrow a}$

- If $m: P \longrightarrow Q$ is a morphisms (i.e., a natural transformation) between two presheaves over $\mathcal{O}(A)$, we define $G_{m} \triangleq m_{A}: P_{A} \rightarrow Q_{A}$. Let us prove that $G_{m}=m_{A}$ is a morphism in $\mathcal{O} f e(A,<)$. Let $x, y \in P_{A}$ such that $x \equiv_{a} y$, that is $P_{i n_{\downarrow a, A}}(x)=P_{i n_{\downarrow a}, A}(y)$; then, by naturality of $m$,

$$
Q_{i n_{\downarrow a, A}}\left(m_{A}(x)\right)=m_{\downarrow a}\left(P_{i n_{\downarrow a, A}}(x)\right)=m_{\downarrow a}\left(P_{i n_{\downarrow a, A}}(y)\right)=Q_{i n_{\downarrow a, A}}\left(m_{A}(y)\right)
$$

an thus $m_{A}(x) \equiv_{a} m_{A}(y)$ in $Q_{A}$.

The above definition is motivated by the fact that $P_{A}$ is the limit of the functor $P$ and that $P_{i n_{\downarrow a, A}}: P_{A} \rightarrow P_{\downarrow a}$ are the relative projections.

The functors $F, G$ form an adjoint pair:

Proposition 1. $\mathcal{O} f e(A,<) \frac{F}{<\frac{\perp}{G}} \widehat{\mathcal{O}(A)}$.

Proof. Let $O=(A,<, X, \equiv)$ be a o.f.e., and $P: \mathcal{O}(A)^{o p} \rightarrow \mathcal{S}$ et a presheaf over $\mathcal{O}(A)$; we prove that $\operatorname{Hom}_{\widehat{\mathcal{O}(A)}}\left(F_{O}, P\right) \cong \operatorname{Hom}_{\mathcal{O} f e(A,<)}\left(O, G_{P}\right)$.

For each natural transformation $m: F_{O} \longrightarrow P$, we can define the corresponding map $f: O \rightarrow G_{P}$ simply as $f: X \rightarrow P_{A}, f(x) \triangleq m_{A}\left([x]_{\equiv_{A}}\right)$.

On the other hand, for each morphism on the $\mathcal{O} f e, f: O \rightarrow G_{P}$ we define the corresponding natural transformation $m: F_{O} \longrightarrow P$ in $\widehat{\mathcal{O}(A)}$ as $m_{U}\left([x]_{\equiv_{U}}\right) \triangleq$ $\left.f(x)\right|_{U}$, for each $U \in \mathcal{O}(A), x \in X$. It is readily proved than $m$ is a natural transformation.

It is easy to check that this bijection is natural in $O$ and $P$, hence the thesis.

Both $F$ and $G$ are neither full nor faithful.

Notice also that $G F \not I d$; in fact $G F: \mathcal{O} f e(A,<) \longrightarrow \mathcal{O} f e(A,<)$ is a quotient operation on the o.f.e.'s. Indeed, if $O=(A,<, X, \equiv)$ is an o.f.e., then $G F_{O}=$ $\left(A,<, X / \equiv_{A}, \equiv^{\prime}\right)$ where $\equiv_{A}=\bigcap_{a \in A} \equiv_{a}$ and $[x] \equiv_{a}^{\prime}[y] \stackrel{\triangle}{\Longleftrightarrow} x \equiv_{\downarrow a} y$.

Finally, it is easy to check that these functors respect the two notions of contractivity on o.f.e.'s and on presheaves. Recall the definition of contractivity on o.f.e.'s from [4]:

Definition 9 (Contractivity on o.f.e.'s). Given an o.f.e. $O=(A,<, X, \equiv)$ a function $f: X \rightarrow X$ is contractive if for every pair of elements $x, y \in X$ and for every element $a$ in $A$, if $\forall a^{\prime}<a . x \equiv_{a^{\prime}} y$, then $f(x) \equiv_{a} f(y)$. 
Then, contractive maps on o.f.e.'s correspond to contractive natural transformations on presheaves:

Proposition 2. (i) Let $O=(A,<, X, \equiv)$ be an o.f.e., and let $f: O \rightarrow O$ be a contractive morphism. Then, $F_{f}: F_{O} \rightarrow F_{O}$ is a contractive natural transformation in $\widehat{\mathcal{O}(A)}$.

(ii) Let $m: P \longrightarrow P$ be a contractive natural transformation in $\widehat{\mathcal{O}(A)}$. Then, $G_{m}: G_{P} \rightarrow G_{P}$ is a contractive morphism in $\mathcal{O} f e(A,<)$.

\subsection{Complete Ordered Families of Equivalences and Sheaves}

The general fixed point theorem of [4] applies to o.f.e.'s where all "Cauchy-like" successions have limits. We recall the following definitions from [4]:

Definition 10. Let $O=(A,<, X, \equiv)$ be an o.f.e., $I$ a subset of $A$, and $\left(x_{a}\right)_{a \in I}$ a family of elements in $X$, indexed by $I$.

- We say that $\left(x_{a}\right)_{a \in I}$ is coherent if $\forall a^{\prime}, a \in I . a^{\prime}<a \Longrightarrow x_{a^{\prime}} \equiv_{a^{\prime}} x_{a}$.

- We say that $\left(x_{a}\right)_{a \in I}$ has as a limit $y$ if $\forall a^{\prime} \in I . x_{a^{\prime}} \equiv_{a^{\prime}} y$.

Definition 11. A complete ordered family of equivalences (c.o.f.e.) is a tuple $O=\left(A,<, X, \equiv, \lim _{. \in A}, \lim _{.<\cdot}\right)$ such that

$-(A,<, X, \equiv)$ is an o.f.e.;

- lim. $\in A$ is a function such that for all coherent families $\left(x_{a}\right)_{a \in A}, \lim _{a \in A} x_{a}$ is a limit for $\left(x_{a}\right)_{a \in A}$;

- $\lim .<$. is a function 2 such that for all $a \in A$ and for all coherent families $\left(x_{a^{\prime}}\right)_{a^{\prime} \in \downarrow a}$ : $\lim _{a^{\prime}<a} x_{a^{\prime}}$ is a limit for $\left(x_{a^{\prime}}\right)_{a^{\prime} \in \downarrow a}$.

For each well-founded order $(A,<)$, the complete o.f.e.'s over it and their morphisms as o.f.e.'s form a category denoted by $\mathcal{C}$ ofe $(A,<)$.

In this definition of c.o.f.e.'s we explicitly require the existence of two functions providing the limits for each coherent family. However, in order to establish an adjuctions with the category of sheaves we need to adopt a weaker notion of completeness, where we only require existence of limits without asking for the limit functions:

Definition 12. A weakly complete ordered family of equivalences is a tuple $O=(A,<, X, \equiv)$ which is an o.f.e. and

- every coherent family of the form $\left(x_{a}\right)_{a \in A}$ has a limit,

- for each $a \in A$, every coherent family of the form $\left(x_{b}\right)_{b \in \downarrow a}$ have a limit.

For each well-founded order $(A,<)$, we denote by $w \mathcal{C}$ ofe $(A,<)$ the full subcategy of $\mathcal{O} f e(A,<)$ whose objects are weakly complete o.f.e.'s.

${ }^{2}$ Using a "dependent type" notation, the arity of the two limit constructors is $\lim . \in A$ : $(A \rightarrow X) \rightarrow X$ and $\lim _{.<.}: \prod_{a \in A}(\downarrow a \rightarrow X) \rightarrow X$. 
Clearly, one can define a forgetful functor $U: \mathcal{C}$ ofe $(A,<) \hookrightarrow w \mathcal{C}$ ofe $(A,<)$ and there is an immersion functor $w \mathcal{C}$ ofe $(A,<) \hookrightarrow \mathcal{O} f e(A,<)$. Moreover, the category $w \mathcal{C}$ ofe $(A,<)$ is connected also to a suitable subcategory of the category of sheaves $\operatorname{Sh}(A)$ over the topological space $(A, \mathcal{O}(A))$, as we will see next.

Let $\mathcal{E}$ be the full subcategory of $\mathrm{Sh}(A)$ of epi-preserving functors, i.e. of sheaves $S$ such that for all $U \subseteq V \in \mathcal{O}(A): S_{i n_{U, V}}: S_{V} \rightarrow S_{U}$ is ep/3. Clearly we have the inclusions $\mathcal{E} \hookrightarrow \operatorname{Sh}(A) \hookrightarrow \widehat{\mathcal{O}(A)}$. We will show next that the adjunction of Proposition 11 can be lifted along the two inclusions $w \mathcal{C}$ ofe $(A,<) \hookrightarrow \mathcal{O} f e(A,<)$ and $\mathcal{E} \hookrightarrow \widehat{\mathcal{O}(A)}$, by proving that $F$ and $G$ maps weakly complete o.f.e.'s in sheaves and sheaves in weakly complete o.f.e.'s, respectively.

Let us prove that for $O=(A,<, X, \equiv)$ a weakly complete o.f.e., the presheaf $S \triangleq F_{O}$ is indeed a sheaf. Let $U$ be an open subset of $A$, and $U=\bigcup_{i \in I} U_{i}$ be an open covering of $U$. Let $\left\{s_{i} \in S_{U_{i}}\right\}_{i \in I}$ be a family of pairwise compatible sections; more explicitly, for all $i, j \in I$, we have that $s_{i} \in X / \equiv_{U_{i}}, s_{j} \in X / \equiv_{U_{j}}$ and $S\left(i n_{i}\right)\left(s_{i}\right)=S\left(i n_{j}\right)\left(s_{j}\right)$ where $i n_{i}: U_{i} \cap U_{j} \subseteq U_{i}$ and $i n_{j}: U_{i} \cap U_{j} \subseteq U_{j}$. We have to define a unique amalgamation of these sections, that is a unique $s \in S_{U}$ such that for all $i \in I: S($ in $)(s)=s_{i}$, where in : $U_{i} \subseteq U$.

For each $a \in U$, there exists an open set $U_{i}$ in the covering of $U$ such that $a \in U_{i}$. Let $s_{a} \triangleq S\left(i n_{a}\right)\left(s_{i}\right) \in S_{\downarrow a}=X / \equiv_{\downarrow a}$ (where $i n_{a}: \downarrow a \subseteq U_{i}$ ); this definition is well given because it does not depend on the particular $U_{i}$ we choose, since the sections are pairwise compatible. For each $a$ in $U$, therefore, let us choose a representant $x_{a} \in s_{a}$ of the equivalence class $s_{a}$; we get thus a family $\left(x_{a}\right)_{a \in U}$ which is coherent (again for the compatibility of the sections). Therefore, since $\mathcal{O}$ is a weakly complete o.f.e. and for the arguments used in [4, Prop. 1], there exists the limit $x$ for the coherent family $\left(x_{a}\right)_{a \in U}$. We can define the amalgamation $s$ as $s \triangleq[x]_{\equiv_{U}}$. This amalgamation is unique: let $s^{\prime} \in S_{U}=X / \equiv_{U}$ be another partition such that for all $i \in I:\left.s^{\prime}\right|_{U_{i}}=s_{i}$ in $X / \equiv_{U_{i}}$. Let $y \in s^{\prime}$; since $\left.s^{\prime}\right|_{U_{i}}=S_{i n_{U_{i}, U}}\left(s^{\prime}\right)$, this means that for all $i \in I:[y]_{\equiv_{U_{i}}}=[x]_{\equiv_{U_{i}}}$, i.e. $y \equiv_{U_{i}} x$. Since $\equiv_{U}=\bigcap_{i \in I} \equiv_{U_{i}}$, we have that $y \equiv_{U} x$, and hence $s^{\prime}=[y]_{\equiv_{U}}=[x]_{\equiv_{U}}=s$.

On the other hand, we prove that the functor $G$ maps epi-preserving sheaves to weakly complete o.f.e.'s. Let $S: \mathcal{O}(A)^{o p} \rightarrow \mathcal{S}$ et be a sheaf in $\mathcal{E}$, we prove that the o.f.e. $G_{S}=\left(A,<, S_{A}, \equiv\right)$ has all the required limits. Let $a \in A$ and let $\left(x_{b}\right)_{b \in\rfloor a}$ be a coherent family in $S_{A}$. To each $x_{b}$ we associate an element $s_{b} \triangleq$ $S_{i n_{\downarrow b}, A}\left(x_{b}\right) \in S_{\downarrow b}$. It is immediate to see that this family $\left\{s_{b}\right\}$ forms a pairwise compatible section, and that $\bigcup_{b \in \downarrow a} \downarrow b=\mathrm{p} \downarrow a=\downarrow a$. Therefore, there exists a (unique) amalgamation $s \in S_{\downarrow^{a}}$. Since $S$ is epi-preserving, $S_{i n_{\downarrow_{a}, A}}: S_{A} \rightarrow S_{\downarrow^{a} a}$ is epi, and therefore there exists an $x \in S_{A}$ such that $\left.x\right|_{\downarrow_{a}}=s$. This means that for all $b \in\rfloor a:\left.x\right|_{\downarrow b}=s_{b}=\left.x_{b}\right|_{\downarrow b}$, that is $x \equiv_{b} x_{b}$, as required.

A similar (and simpler) argument shows that any coherent family of the form $\left(x_{a}\right)_{a \in A}$ has limit. We have thus proved the following

Proposition 3. $w \mathcal{C}$ ofe $(A,<) \underset{<\frac{\perp}{G}}{\stackrel{F}{<}} \mathcal{E}$

$\overline{{ }^{3} \text { Each } i n_{U, V}}: V \rightarrow U$ in $\mathcal{O}(A)^{o p}$ is epi because $i n_{U, V}: U \hookrightarrow V$ is mono in $\mathcal{O}(A)$. 
The diagram aside summarizes the relationships between the categories introduced in this paper. The functor a : $\widehat{\mathcal{O}(A)} \longrightarrow \mathrm{Sh}(A)$, left adjoint of the inclusion functor $i$, is the associated sheaf functor. Intuitively, $\mathbf{a}(P)$ is the "closest" sheaf to the presheaf $P$, i.e., the "best approximation" of $P$ where the GFPT can be applied.

This diagram points out also the GFPT over $\operatorname{Sh}(A)$ is strictly stronger than the previous result over c.o.f.e.'s [4 Theorem 1]. Indeed the latter can be derived as a corollary of the former:

Corollary 1 (GFPT for c.o.f.e.'s). Let $C=\left(A,<, X, \equiv, \lim _{. \in A}, \lim _{.<.}\right)$be $a$ c.o.f.e., and $f: C \rightarrow C$ a contractive map. Then, there exists $x \in X$ such that $f(x) \equiv_{A} x$. Moreover, for all $y \in X$ such that $f(y) \equiv_{A} y$, we have that $x \equiv_{A} y$.

Proof. By applying the forgetful functor, $U(C)=(A,<, X, \equiv)$ is a weakly complete o.f.e., and $f$ is a contractive map on $U(C)$. For Proposition[3, $S \triangleq F(U(C))$ is an epi-preserving sheaf, and in particular it is a sheaf in $\operatorname{Sh}(A)$. By Proposition 2, $F(f): S \longrightarrow S$ is contractive, and therefore, by Theorem 1 , there exists a unique natural transformation $\mu: 1 \rightarrow S$ such that $F(f) \circ \mu=\mu$. In particular, this means that $\mu_{A}: 1 \rightarrow S$ is a partition class (i.e., $\mu_{A} \in X / \equiv_{A}$ ) such that $F(f)_{A}\left(\mu_{A}\right)=\mu_{A}$. By definition of $F$, this means that for all $x \in \mu_{A}$ : $[f(x)]_{\equiv_{A}}=\mu_{A}=[x]_{\equiv_{A}}$, that is, $f(x) \equiv_{A} x$. If there is another $y \in X$ such that $f(y) \equiv_{A} y$, then $[y]_{\equiv_{A}}=[f(y)]_{\equiv_{A}}=[f(x)]_{\equiv_{A}}$ (by uniqueness of $m$ ), and therefore $[y]_{\equiv_{A}}=[x]_{\equiv_{A}}$, that is $x \equiv_{A} y$.

\section{Conclusions}

In this paper we have presented a novel approach to the problem of establishing fixed points of circular definitions. Our approach, based on categories of sheaves over "well-founded" topologies, unifies the well-founded induction principle and a generalization of Banach theorem. This result encompasses most known techniques used in Mathematics and Computer Science for ensuring convergency of circular definitions, and moreover it can be applied also to definitions which are both recursive and corecursive at the same time.

Our work has been motivated also by the need of understanding better and generalize a similar set theoretic result, based on the notion of "(complete) ordered family of equivalences" [4]. In fact, we have shown that c.o.f.e.'s can be seen as a particular case of sheaves on well-founded topologies, suited for implementation in proof assistants. However, the generality we have achieved in this paper goes well beyond this; for instance, the new model offers a cleaner and more elegant treatment of the important case of functions defined by wellfounded recursion. 
Future work. As pointed out before, the theory of sheaves over well-founded topologies presented in this paper is stronger than the theory of c.o.f.e.'s implemented in Coq (and the similar development in Isabelle/HOL). A way for gaining this expressive power could be to formalize directly the theory presented in this paper as it is; however, such a formalization would be quite different from the one of c.o.f.e.'s - and likely not as easy to use. Another possibility would be to look for a counterpart of $\operatorname{Sh}(A)$ within the category of o.f.e.'s. In other words, we conjecture the existence of a reflective subcategory $\mathcal{C} \hookrightarrow \mathcal{O} f e(A,<)$, such that $\mathcal{C}$ ofe $(A,<) \hookrightarrow \mathcal{C}$ and connected to $\operatorname{Sh}(A)$ by the adjunction $F \dashv G$. This would complete the diagram above, and would lead to a compact, implementationoriented representation of sheaves.

In this paper, we have considered presheaves and sheaves over a precise kind of topology, namely the one formed by downward closed sets. It is interesting future work to understand at what extent the notions and results of this paper can be generalized to other topologies. For instance, the key notion of "wellfounded topology" should be readily adapted to general Grothendieck topologies.

Another interesting future work is to consider the internal language of the category $\operatorname{Sh}(A)$. As for any topos, $\operatorname{Sh}(A)$ supports directly the interpretation of a typed (intuitionistic) higher order logic, but moreover, we should be able to extend this language with specific constructors and rules for fixpoint definition.

\section{References}

1. A. Balaa and Y. Bertot. Fix-point equations for well-founded recursion in type theory. In Proc. TPHOL 2000, LNCS 1869, pages 1-16. Springer-Verlag, 2000.

2. A. Bove. General recursion in type theory. In [5], pages 39-58.

3. J. W. de Bakker and J. I. Zucker. Processes and the denotational semantics of concurrency. Information and Control, 54:70-120, 1982.

4. P. Di Gianantonio and M. Miculan. A unifying approach to recursive and corecursive definitions. In [5], pages 148-161.

5. H. Geuvers and F. Wiedijk, editors. Proceedings of TYPES'02, LNCS 2646. Springer-Verlag, 2003.

6. E. Giménez. Codifying guarded recursion definitions with recursive schemes. In Proc. of TYPES'94, LNCS 996, pages 39-59, 1995. Springer-Verlag.

7. E. Gimenez. A tutorial on recursive types in Coq. RT-0221, INRIA, 1998.

8. INRIA. The Coq Proof Assistant, 2003. http://coq.inria.fr/doc/main.html .

9. J. L. Kelley. General Topology. Springer-Verlag, 1975.

10. J. Lambek and P. J. Scott. Introduction to higher order categorical logic, volume 7 of Cambridge studies in advanced mathematics. Cambridge University Press, 1986.

11. J. Matthews. Recursive function definition over coinductive types. In Proc. TPHOL'99, LNCS 1690, pages 73-90. Springer-Verlag, 1999.

12. B. Nordström. Terminating general recursion. BIT, 28:605-619, 1988.

13. C. Paulin-Mohring. Inductive definitions in the system Coq; rules and properties. In Proc. of TLCA'93, LNCS 664, pages 328-345. Springer-Verlag, 1993. 